УДК 004.932

А. С. РУБЕЛЬ, В. В. ЛУКИН

Национальный аэрокосмический университет им. Н. Е. Жуковского «ХАИ», Украина

\title{
АНАЛИЗ И ПРОГНОЗИРОВАНИЕ ЭФФЕКТИВНОСТИ ФИЛЬТРАЦИИ \\ С ИСПОЛЬЗОВАНИЕМ БЕЗЭТАЛОННЫХ МЕР ВИЗУАЛЬНОГО КАЧЕСТВА ИЗОБРАЖЕНИЙ
}

\begin{abstract}
Проведен анализ эффективности и иелесообразности применения фильтрации с использованием безэталонных мер визуального качества изображений. Также рассмотрены возможные способы прогнозирования субъективных оценок визуального качества изображений после применения фильтрации на основе одного или нескольких входных параметров. Анализ выполнен для ДКП и BMЗD-фильтров. C помощью спеииализированной базы тестовых изображений SubjectiveIQA проанализировано соответствие восприятию человека существующих безэталонных мер визуального качества для отфильтрованных изображений. Показано, что коэффициент ранговой корреляции Спирмена между значениями безэталонных мер и субъективными оченками не превымает 0,7.
\end{abstract}

Ключевые слова: эффективность фильтрачии, безэталонные меры визуального качества изображений, прогнозирование, ДКП фильтр, ВМЗD, аддитивные помехи, субъективный эксперимент.

\section{Введение}

Фильтрация изображений является одним из ключевых этапов в предварительной обработке изображений [1]. Считается, что применение фильтрации позволяет не только улучшить визуальное качество изображений, но и повысить эффективность последующих этапов обработки, таких как сжатие, сегментация, классификация, изменение размеров объектов и распознавание [1].

За последние десятилетия было разработано огромное количество фильтров, начиная от простой линейной фильтрации и заканчивая сложными методами на основе поиска подобных блоков [2-5] и глубоких нейронных сетей $[6,7]$. При таком разнообразии фильтров вполне естественно возникает множество вопросов. Два основных из них - какой фильтр обеспечивает лучшее визуальное качество и насколько целесообразно применять фильтрацию? Несмотря на то, что разработка новых методов фильтрации изображений ведется достаточно интенсивно продолжительное время, лишь незначительное количество работ посвящено разработке и верификации методов оценки визуального качества изображений после применения фильтрации [8-12]. Не имея в своем распоряжении надежных мер качества, невозможно адекватно сравнивать существующие методы фильтрации.

Несмотря на многие преимущества, практически все существующие фильтры имеют тенденцию к чрезмерному сглаживанию границ изображения, мелких деталей и текстур [11-13]. В связи с этим одновременно с подавлением шума теряются ин- формативные признаки изображения, и фильтрация может не приводить к улучшению визуального качества, фактически становясь бесполезной или даже вредной. Вследствие этого необходимо иметь возможность адекватно оценивать качество отфильтрованного изображения и, следовательно, принимать решение о целесообразности применения фильтрации.

Визуальное качество изображения может быть оценено как объективными мерами визуального качества [14], так и субъективными методами $[9,10]$. Субъективные методы основаны на получении мнений (Mean Opinion Score, MOS) от людей, привлеченных к оцениванию качества изображений. Эти методы являются основополагающими при разработке алгоритмов визуального восприятия человеком качества изображений. По этой причине субъективные методы считаются наиболее надежными подходами к оцениванию визуального качества отфильтрованных изображений. С другой стороны, данные методы требуют много времени на получение достаточного количества мнений и не могут быть использованы в режиме реального времени. В свою очередь, объективные меры качества направлены на разработку моделей для автоматического и точного прогнозирования усредненных субъективных оценок визуального качества людьми (MOS).

На данный момент разработано большое количество различных объективных мер визуального качества изображений $[8-10,15-19]$. Однако особый интерес представляют меры визуального качества при отсутствии эталона [18]. Это связано с широким кругом задач, когда эталонное (без шума) изображе- 
ние отсутствует и необходимо оценивать качество при наличии лишь искаженного изображения. Таковыми задачами являются слепая оценка параметров шума, эффектов размытия, уровня блочных артефактов при сжатии изображений. В данной работе основное внимание уделено искажениям, возникающим при фильтрации изображений, исходно искаженных шумом.

Отметим, что субъективные эксперименты по оцениванию визуального качества изображений после применения фильтрации с различным количеством участников уже были выполнены нами ранее $[12,20]$. В этих экспериментах были рассмотрены два фильтра - стандартный ДКП фильтр [4] и фильтр BM3D [3], являющийся на данный момент одним из наилучших фильтров. В результате для каждого тестового изображения, уровня помех и фильтра были получены вероятности того, что отфильтрованное изображение воспринимается людьми как имеющее лучшее визуальное качество, чем соответствующее исходное (нефильтрованное) изображение. Так, например, значение вероятности больше 0,6 означает, что применять фильтрацию целесообразно. Если же вероятность находится в интервале от 0,5 до 0,6 , то можно сказать, что использование фильтрации имеет смысл. В противном случае применять фильтрацию не стоит.

Было также показано [12], что фильтрация, в основном, целесообразна для шума средней интенсивности и изображений с довольно простой структурой. Для текстурных изображений или изображений, содержащих много мелких деталей, фильтрация не приводит к улучшению визуального качества обработанного изображения по сравнению с исходным (искаженным) [12, 13, 20].

На практике исследователи часто используют стандартные меры, такие как пиковое отношение сигнал-шум (PSNR) и структурное подобие (SSIM) [15] для сравнения качества изображений и алгоритмов фильтрации, однако адекватность этих мер находится под большим сомнением [9]. Для других мер визуального качества с эталоном также имеются проблемы [8-10]. Напомним, что ранее были предложены методы прогнозирования эффективности фильтрации, позволяющие предсказать выигрыш для различных мер визуального качества с эталоном с довольно высокой точностью для нескольких современных методов фильтрации [13, 21, 22]. Однако такой формальный выигрыш не всегда свидетельствует о положительном эффекте от фильтрации, особенно при обработке текстурных изображений $[12$, $13,20]$.

Поэтому возникает вопрос о применимости безэталонных мер визуального качества. Существующие безэталонные меры визуального качества не были тщательно исследованы для анализа качества изображений после применения фильтрации. Отметим, что обычно предполагается, что чем выше выигрыш для заданной меры (т.е. разность между значениями меры на выходе и входе фильтра), тем лучше визуальное качество обработанного изображения.

Ввиду этого желательно также прогнозировать субъективные оценки визуального качества (MOS) отфильтрованных изображений. Эта информация может помочь понять, когда фильтрация целесообразна. Заметим, что предварительной анализ такого прогнозирования на основе мер качества при наличии эталона (PSNR и PSNR-HVS-M [16]) показал недостаточно высокую точность [23]. Было показано, что корреляция между рассматриваемыми мерами и вероятностями предпочтения фильтрованных изображений исходным составляет порядка 0,2 .

Целью данной работы является анализ целесообразности применения фильтрации с использованием безэталонных мер визуального качества изображений. Кроме того, в работе рассматриваются возможные способы прогнозирования субъективных оценок визуального качества отфильтрованных изображений на основе одного или нескольких входных параметров (безэталонных мер).

\section{Описание базы тестовых изображений и субъективных экспериментов}

Кратко рассмотрим проведенные ранее эксперименты и их результаты. В экспериментах использовалась база из 16 тестовых изображений, представленных в оттенках серого. Восемь из них были взяты из базы TID2013 [9], остальные изображения являются полностью текстурными [24]. Примеры тестовых изображений приведены на рис. 1.

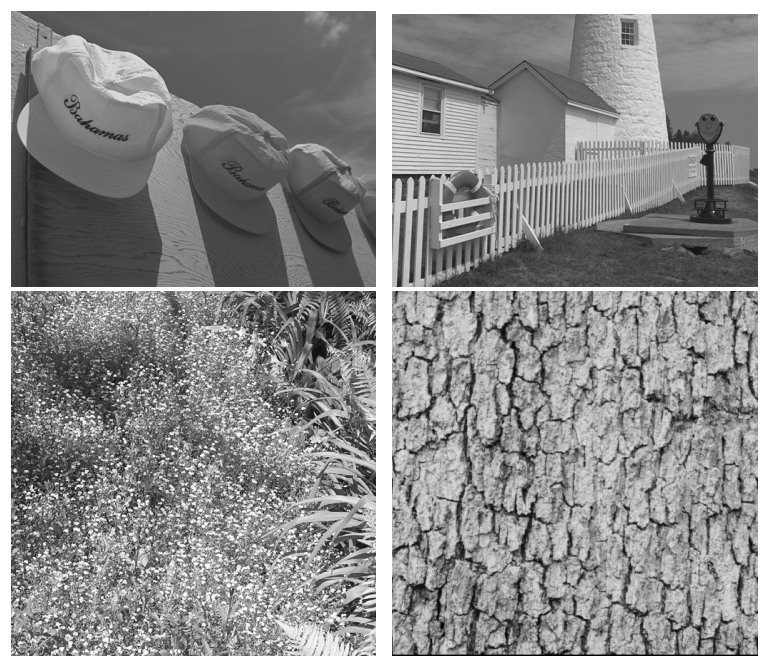

Рис. 1. Примеры тестовых изображений 
Каждое тестовое изображение, искаженное аддитивным белым гауссовым шумом с нулевым средним и варьируемым среднеквадратическим отклонением (СКО) было обработано двумя фильтрами - ДКП фильтром и BM3D [12, 20]. Значения СКО шума были равны 3, 5, 10, 15, 20, 25 и 30. Таким образом, база состояла из 112 зашумленных и 224 отфильтрованных изображений.

Эксперимент проводился следующим образом. На экране одновременно показывались два изображения - зашумленное и после фильтрации. Каждый участник должен был выбрать, какое из изображений имеет лучшее визуальное качество. Внешний вид программы, используемой в экспериментах, показан на рис. 2.

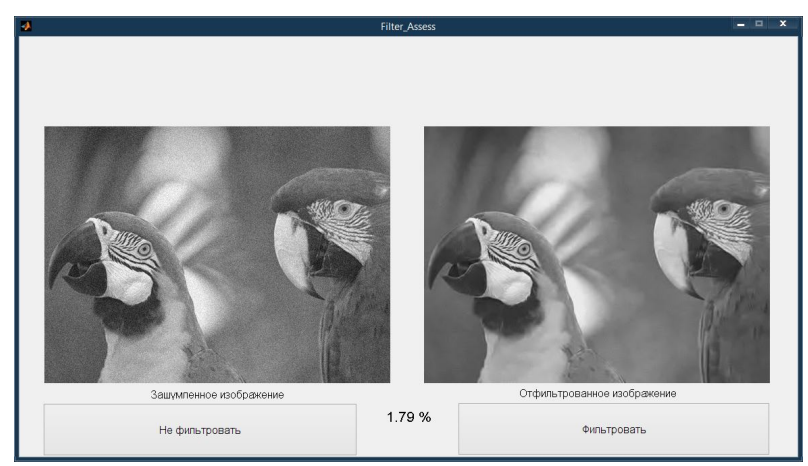

Рис. 2. Внешний вид программы

В результате проведения субъективных экспериментов оказалось возможным для каждого изображения, типа фильтра и СКО шума определить вышеупомянутые вероятности предпочтения фильтрации. Полученные вероятности можно рассматривать как усредненные экспертные оценки (MOS). Более детальный анализ полученных результатов представлен в работе [12].

Общее число добровольцев, принявших участие в субъективных экспериментах, составляет 109 человек. База тестовых изображений и экспериментальные данные доступны онлайн https://github.com/ViA-RiVaL/SubjectiveIQA.

\section{Применение базы тестовых изображений для верификации безэталонных мер визуального качества}

Применим рассмотренную выше базу тестовых изображений для оценки эффективности существующих безэталонных мер визуального качества. Полученная эффективность позволит нам определить, насколько та или иная мера качества соответствует восприятию человека. Эффективность меры качества определяется ранговой корреляцией между значениями MOS (в нашем случае это вероятности предпочтения фильтрации $\mathrm{P}_{\text {pref }}$ ) и значениями безэталонных мер. В качестве корреляции используются ранговые корреляции Спирмена и Кендалла. Отметим, что оценка эффективности проводилась между значениями выигрыша для рассматриваемых мер (т.е. разностями между значениями меры для отфильтрованного и зашумленного изображений) и вероятностями предпочтения фильтрации $\mathrm{P}_{\text {pref }}$. Использование ранговой корреляции обусловлено тем, что в рассматриваемом случае линейность зависимости между мерой визуального качества и $\mathrm{P}_{\text {pref }}$ не обязательна.

В данной работе была осуществлена оценка адекватности широкого круга современных безэталонных мер качества: BRISQUE [25], SSEQ [26], BIQI [27], BLIINDS-II [28], CORNIA [29], NIQE [30], JNB [31], OG-IQA [32], M3 [33], DIIVINE [34], TCLT [35], NFERM [36], LPSI [37], QAC [38], CPBD [39], NR-PWN [40], dipIQ [41], HOSA [42], Naturalness Factor (NF) [43], SR [44], SISBLIM [45].

B табл. 1 приведены результаты ранговой корреляции Спирмена между значениями выигрыша для рассматриваемых безэталонных мер и $\mathrm{P}_{\text {pref }}$ для двух анализируемых фильтров.

Таблица 1

Меры визуального качества без эталона

\begin{tabular}{|c|c|c|}
\hline \multirow[t]{2}{*}{ Меры } & \multicolumn{2}{|c|}{ Значения корреляции } \\
\hline & ДКП & BM3D \\
\hline BRISQUE [25] & 0,0099 & 0,4265 \\
\hline SSEQ [26] & 0,2721 & 0,6111 \\
\hline BIQI [27] & 0,0182 & 0,3501 \\
\hline BLIINDS-II [28] & 0,0459 & 0,339 \\
\hline CORNIA [29] & 0,2247 & 0,5942 \\
\hline NIQE [30] & 0,0364 & 0,4391 \\
\hline JNB [31] & 0,0395 & 0,3242 \\
\hline OG-IQA [32] & 0,0848 & 0,4517 \\
\hline M3 [33] & 0,3516 & 0,0615 \\
\hline DIIVINE [34] & 0,1841 & 0,4984 \\
\hline TCLT [35] & 0,0781 & 0,2137 \\
\hline NFERM [36] & 0,1291 & 0,4274 \\
\hline LPSI [37] & 0,4076 & 0,0381 \\
\hline QAC [38] & 0,1949 & 0,2108 \\
\hline CPBD [39] & 0,0767 & 0,3281 \\
\hline NR-PWN [40] & 0,2197 & 0,2083 \\
\hline dipIQ [41] & 0,0981 & 0,4666 \\
\hline HOSA [42] & 0,0917 & 0,5423 \\
\hline NF [43] & 0,3306 & 0,6023 \\
\hline SR [44] & 0,022 & 0,2996 \\
\hline SISBLIM_SM [45] & 0,1767 & 0,2486 \\
\hline SISBLIM_SFB [45] & 0,158 & 0,2947 \\
\hline SISBLIM_WM [45] & 0,3489 & 0,0938 \\
\hline SISBLIM_WFB [45] & 0,3337 & 0,1093 \\
\hline
\end{tabular}




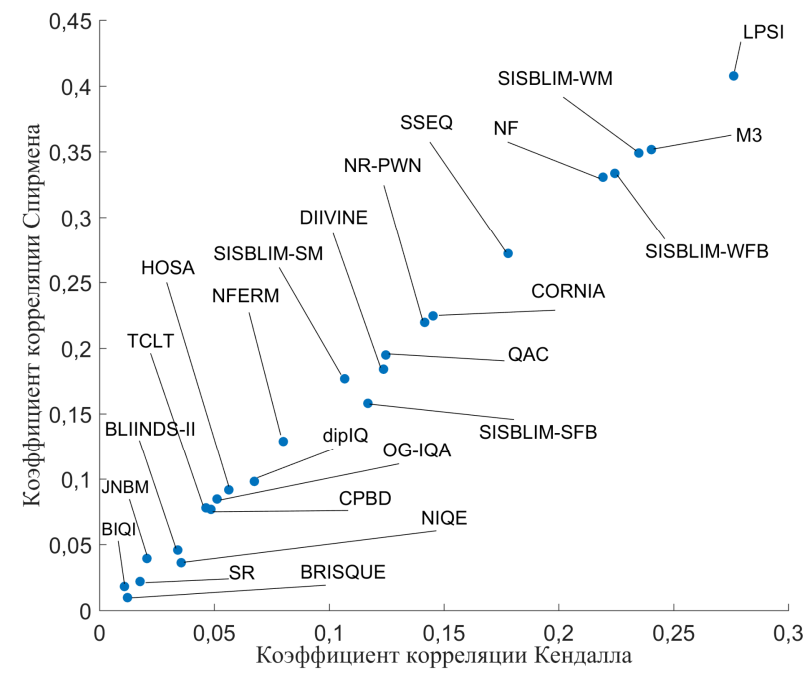

a

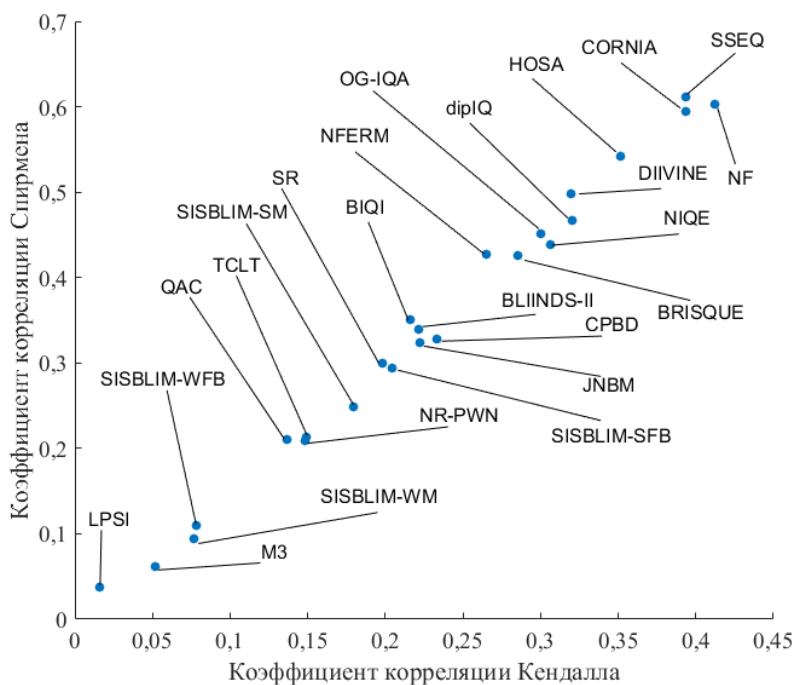

6

Рис. 3. Результаты ранговых корреляций Спирмена и Кендалла между значениями выигрыша для рассматриваемых безэталонных мер качества и $\mathrm{P}_{\text {pref }}$ для ДКП фильтра (а) и BM3D фильтра (б)

На рис. 3 совместно представлены результаты анализа эффективности рассматриваемых безэталонных мер для обоих фильтров. Здесь, горизонтальные и вертикальные оси соответствуют значениям ранговых корреляций Кендалла и Спирмена. Наилучшими являются те меры, для которых соответствующие точки расположены ближе к верхнему правому углу.

Как видно из представленных результатов (см. табл. 1), даже самая лучшая мера лишь весьма умеренно коррелирует с субъективными оценками визуального качества отфильтрованных изображений. Можно заметить, что значения корреляции для изображений, обработанных ДКП фильтром, несколько меньше, чем для BM3D-фильтра. Также из рис. 3 хорошо видно, что меры LPSI [37] и M3 [33] имеют наибольшие значения корреляции для ДКП фильтра, однако для BM3D фильтра наилучшими являются меры SSEQ [26], NF [43] и CORNIA [29].

Приведенные выше результаты показывают, что все рассмотренные безэталонные меры качества плохо соответствуют восприятию человеком отфильтрованных изображений. Это говорит о том, что база изображений [46] может эффективно использоваться для совершенствования существующих безэталонных мер и тестирования новых мер визуального качества.

\section{Анализ возможностей прогнозирования эффективности фильтрации}

Теперь перейдем к рассмотрению возможных способов прогнозирования субъективных оценок визуального качества отфильтрованных изображений на основе безэталонных мер. Напомним, что в нашем случае субъективные оценки представляют собой вероятность предпочтения отфильтрованных изображений исходным (зашумленным).

Идея прогнозирования субъективных оценок состоит в следующем [13, 21, 22, 23]. Предполагается, что существует параметр, который адекватно характеризует эффективность фильтрации, а затем этот параметр используется для принятия решения о целесообразности применения фильтрации. Таковым параметром у нас является упомянутая ранее вероятность предпочтения фильтрации. В работах $[13,21,22]$, посвящённых задаче прогнозирования эффективности фильтрации, такими параметрами выступали меры качества изображений с эталоном. Также есть один или несколько входных параметров, которые описывают обрабатываемое изображение. Предполагается, что существует некая достаточно жесткая зависимость между входными и выходным параметрами. Эта зависимость должна быть получена заранее. Затем (при прогнозировании) вычисляют входной параметр, и он используется в качества аргумента для вычисления выходного параметра, на основании анализа которого и делается вывод о целесообразности применения фильтрации.

Помимо этого, процедура прогнозирования должна быть быстрой (желательно более быстрой, чем сама фильтрация) и достаточно точной, чтобы обеспечить правильность решений, принятых на основе прогноза.

Несмотря на достигнутые ранее положительные результаты прогнозирования эффективности фильтрации $[13,21,22]$ (высокую точность прогно- 
зирования, значения параметра $\mathrm{R}^{2}$, характеризующего точность аппроксимации (вписывания кривой в скаттерограмму) превышают 0,9), следует отметить, что предсказанный выигрыш после применения фильтрации в соответствии с мерами качества не всегда визуально заметен. В связи с этим, для более полного понимания ситуации, когда целесообразно применять фильтрацию, необходимо также прогнозировать субъективную оценку визуального качества отфильтрованного изображения.

Рассмотрим прогнозирование вероятностей предпочтения фильтрации на основе одного параметра, а именно одной из безэталонных мер визуального качества.

Для вписывания кривых нами была выбрана следующая полиномиальная аппроксимирующая функция [21 - 23]:

$$
\mathrm{P}_{\text {pref }}=\sum_{\mathrm{k}=0}^{\mathrm{N}} \mathrm{a}_{\mathrm{k}} \cdot \mathrm{M}^{\mathrm{k}} \text {, }
$$

где $\mathrm{a}_{\mathrm{k}}-\mathrm{k}$-й весовой коэффициент полинома,

M - рассматриваемая безэталонная мера качества.

Точность вписывания кривых при этом оценивается параметром $\overline{\mathrm{R}}^{2}$ (скорректированный коэффициент детерминации) и среднеквадратическим отклонением ошибки (RMSE). Чем выше значения $\overline{\mathrm{R}}^{2}$ и меньше значения RMSE, тем лучше точность прогнозирования.

Характеристики точности прогнозирования $\overline{\mathrm{R}}^{2}$ для различных степеней полинома и обоих фильтров представлены в табл. 2. Отметим, что анализируемые безэталонные меры для каждого фильтра были выбраны в соответствии с их эффективностью (см. результаты в табл. 1).

Как видно из представленных результатов, точность прогнозирования для обоих фильтров невысока. Также можно видеть, что в обоих случаях аппроксимирующие полиномы третьей степени дают наибольшую точность. Стоит отметить также, что точность прогнозирования заметно выше в случае использования фильтра BM3D.

Отметим достигнутый положительный результат - точность прогнозирования для фильтра BM3D несколько улучшилась по сравнению со случаем, когда для прогнозирования использовались меры качества при наличии эталона PSNR и PSNR-HVSM [16] ( $\overline{\mathrm{R}}^{2}$ было около 0,4) [23]. В то же время ситуация с ДКП-фильтром противоположна.

На рис. 4 представлены скаттерограммы с вписанными в них кривыми с использованием аппрок- симирующего полинома третьей степени для значений выигрыша безэталонных мер LPSI и SSEQ для ДКП фильтра и BM3D соответственно.

Отметим, что точки на скаттерограммах были получены с использованием большого количества тестовых изображений, искаженных аддитивным шумом с различной интенсивностью (всего 112 изображений). Для каждого изображения и СКО шума были получены значения выигрыша рассматриваемой безэталонной меры. Значения вероятностей предпочтения фильтрации $\mathrm{P}_{\text {pref }}$ были взяты из субъективных экспериментов.

Таблица 2

Точность прогнозирования

\begin{tabular}{|c|c|c|c|}
\hline Фильтр & \multicolumn{3}{|c|}{ ДКП } \\
\hline \multirow{2}{*}{$\mathrm{N}$} & Mepa & $\overline{\mathrm{R}}^{2}$ & RMSE \\
\hline \multirow{2}{*}{2} & LPSI & 0,1605 & 0,1168 \\
\cline { 2 - 4 } & $\mathrm{M} 3$ & 0,1226 & 0,1194 \\
\hline \multirow{2}{*}{3} & LPSI & 0,1606 & 0,1168 \\
\cline { 2 - 4 } & $\mathrm{M} 3$ & 0,1158 & 0,1199 \\
\hline \multirow{2}{*}{4} & LPSI & 0,1528 & 0,1173 \\
\cline { 2 - 4 } Фильтр & M3 & 0,1086 & 0,1204 \\
\hline \multirow{2}{*}{$\mathrm{N}$} & Mepa & BM3D \\
\hline \multirow{3}{*}{2} & SSEQ & 0,3369 & 0,1242 \\
\cline { 2 - 4 } & NF & 0,3496 & 0,123 \\
\cline { 2 - 4 } & CORNIA & 0,3248 & 0,1253 \\
\hline \multirow{3}{*}{3} & SSEQ & 0,4584 & 0,1122 \\
\cline { 2 - 4 } & NF & 0,3506 & 0,1229 \\
\cline { 2 - 4 } & CORNIA & 0,3378 & 0,1241 \\
\hline \multirow{2}{*}{4} & SSEQ & 0,4567 & 0,1124 \\
\cline { 2 - 4 } & NF & 0,3452 & 0,1234 \\
\cline { 2 - 4 } & CORNIA & 0,3317 & 0,1247 \\
\hline
\end{tabular}

Как видно из анализа скаттерограмм на рис. 4, точки достаточно сильно разбросаны, что и обуславливает низкую точность прогнозирования.

Можно предположить, что совместное использование двух входных параметров может повысить точность прогнозирования. Нами был проведен анализ точности вписывания зависимости $\mathrm{P}_{\text {pref }}$ от значений выигрыша двух безэталонных мер, использованных в качестве входных параметров. Рассматривалось множество возможных вариантов. Ниже даны лучшие из них.

В табл. 3 представлены значения $\overline{\mathrm{R}}^{2}$ и RMSE для двух безэталонных мер для различных степеней аппроксимирующих полиномов.

Из представленных данных явно видно, что двухпараметрическое вписывание не обеспечивает достаточное увеличение точности прогнозирования. 


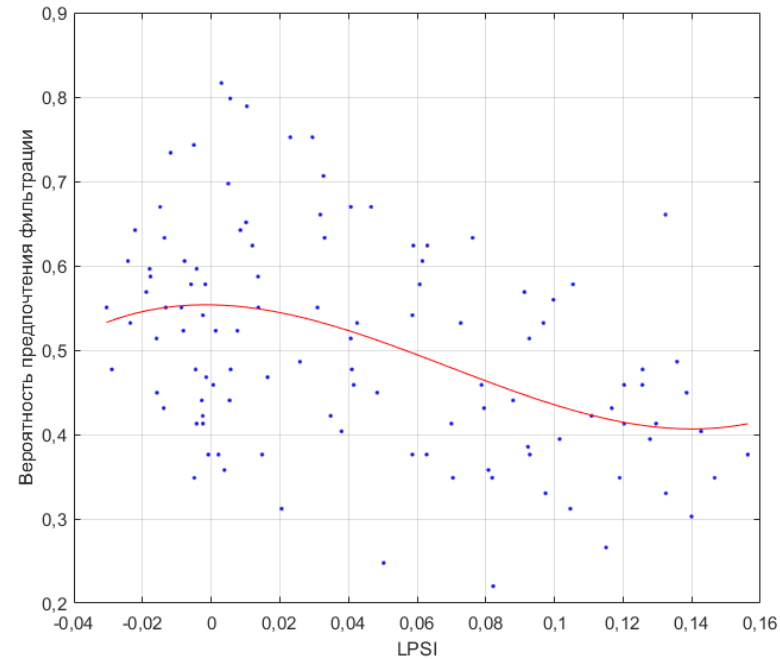

a

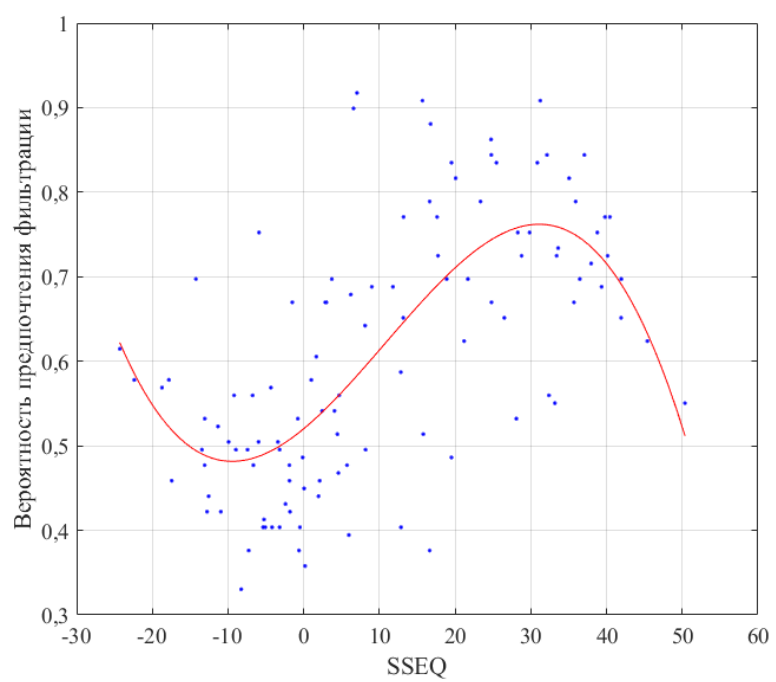

6

Рис. 4. Скаттерограммы и вписанные кривые зависимостей вероятности предпочтения фильтрации от значений выигрыша безэталонных мер LPSI для ДКП фильтра (а) и SSEQ для фильтра BM3D (б)

Таблица 3

Точность прогнозирования

\begin{tabular}{|c|c|c|c|}
\hline Фильтр & \multicolumn{3}{|c|}{ ДКП } \\
\hline $\mathrm{N}$ & Меры & $\overline{\mathrm{R}}^{2}$ & RMSE \\
\hline 2 & \multirow{3}{*}{ LPSI, M3 } & 0,1634 & 0,1166 \\
\hline 3 & & 0,1611 & 0,1168 \\
\hline 4 & & 0,1389 & 0,1183 \\
\hline Фильтр & \multicolumn{3}{|c|}{ BM3D } \\
\hline $\mathrm{N}$ & Меры & $\overline{\mathrm{R}}^{2}$ & RMSE \\
\hline 2 & \multirow{3}{*}{ SSEQ, NF } & 0,4045 & 0,1177 \\
\hline 3 & & 0,5028 & 0,1075 \\
\hline 4 & & 0,4908 & 0,1075 \\
\hline
\end{tabular}

Таким образом, для всех случаев степей аппроксимирующей функции и входных параметров точность прогнозирования вероятности предпочтения фильт- рации по-прежнему остается довольно низкой. На рис. 5 показаны примеры скаттерограмм с вписанными в них поверхностями с использованием аппроксимирующих полиномов третьей степени. Поскольку рассматриваются два входных параметра, аппроксимирующая (регрессионная) зависимость является поверхностью.

Как и ранее, видно, что данные значительно разбросаны.
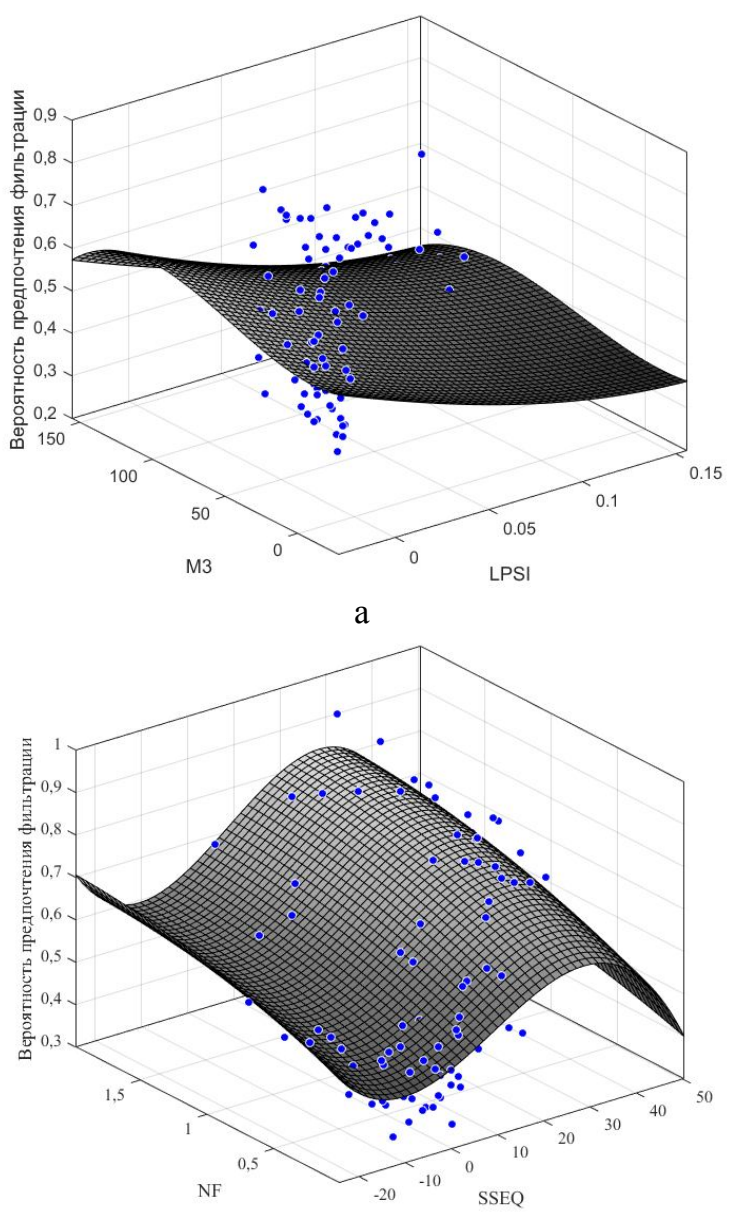

6

Рис. 5. Скаттерограммы и вписанные поверхности зависимостей вероятности предпочтения фильтрации от значений выигрыша для двух мер: M3, LPSI для ДКП фильтра (а) и NF, SSEQ для BM3D (б)

Тем не менее, можно ожидать, что точность прогнозирования может быть повышена за счет использования многопараметрической регрессии и поиска более адекватных (информативных) статистических параметров.

\section{Заключение}

Проанализирована эффективность и целесообразность применения фильтрации с использованием безэталонных мер визуального качества. Рассмотре- 
ны два метода фильтрации - стандартный ДКП фильтр и BM3D. Показано, что современные безэталонные меры визуального качества лишь умеренно коррелируют с субъективными оценками качества отфильтрованных изображений. Определены меры качества, которые являются наилучшими для рассматриваемого приложения. Выполнен предварительный анализ возможных подходов к прогнозированию субъективных оценок качества отфильтрованных изображений на основе безэталонных мер. Показано, что известные безэталонные меры не обеспечивают желаемой точности прогнозирования качества отфильтрованных изображений. Таким образом, задача предсказания субъективной оценки визуального качества отфильтрованных изображений весьма далека от ее решения.

\section{Литература}

1. Schowengerdt, R. Remote Sensing: Models and Methods for Image Processing [Text] / R. Schowengerdt. - Academic Press, 2006. - 560 p.

2. Secrets of image denoising cuisine [Text] / M. Lebrun, M. Colom, A. Buades, J. M. Morel // Acta Numerica. - 2012. - Vol. 21, no. 1. - P. 475-576.

3. Image denoising by sparse $3 D$ transformdomain collaborative filtering [Text] / K. Dabov, A. Foi, V. Katkovnik, K. Egiazarian // IEEE Transactions on Image Processing. - 2007. - Vol. 16, no. 8. - P. 20802095.

4. Image filtering based on discrete cosine transform [Text] / V. Lukin, R. Oktem, N. Ponomarenko, K. Egiazarian // Telecommunications and Radio Engineering. - 2007. - Vol. 66, no. 18. - P. 1685-1701.

5. Pižurica, A. Image Denoising Algorithms: From Wavelet Shrinkage to Nonlocal Collaborative Filtering [Text] / A. Pižurica // Wiley Encyclopedia of Electrical and Electronics Engineering. - 2017. - 17 p.

6. Beyond a Gaussian Denoiser: Residual Learning of Deep CNN for Image Denoising [Text] / K. Zhang, W. Zuo, Y. Chen, D. Meng, L. Zhang // IEEE Transactions on Image Processing. - 2017. - Vol. 26, no. 7. - P. 3142-3155.

7. Fast, Trainable, Multiscale Denoising [Text] / S. Choi, J. Isidoro, P. Getreuer, P. MIlanfar // ArXiv preprint arXiv: 1802.06130. - 2018. -5 p.

8. Zheng, K. Perceptual evaluation of image denoising algorithms [Text] / K. Zheng, Z. Wang // Asilomar Conference on Signals, Systems and Computers, Pacific Grove, CA. - 2013. - P. 1351-1355.

9. Image database TID2013: Peculiarities, results and perspectives [Text] / N. Ponomarenko, L. Jin, O. Ieremeiev, V. Lukin, K. Egiazarian, J. Astola, B. Vozel, K. Chehdi, M. Carli, F. Battisti, C.-C. Jay Kuo //
Signal Processing: Image Communication. - 2015. Vol. 30. - P. 57-77.

10. Statistical evaluation of visual quality metrics for image denoising [Text] / K. Egiazarian, M. Ponomarenko, V. Lukin, O. Ieremeiev // ArXiv preprint arXiv: 1711.00693. - 2017. -5 .

11. HVS-based local analysis of denoising efficiency for DCT-based filters [Text] / O. Rubel, N. Ponomarenko, V. Lukin, J. Astola, K. Egiazarian // Second International Scientific-Practical Conference Problems of Infocommunications Science and Technology (PIC S\&T), Kharkiv, Ukraine. - Oct. 2015. - P. 189-192.

12. Rubel, A. Analysis of visual quality for denoised images [Text] / A. Rubel, O. Rubel, V. Lukin // 14th International Conference The Experience of Designing and Application of CAD Systems in Microelectronics (CADSM), Lviv, Ukraine. - 2017. - P. 92-96.

13. Is Texture Denoising Efficiency Predictable? [Text] / O. Rubel, V. Lukin, S. Abramov, B. Vozel, O. Pogrebnyak, K. Egiazarian // International Journal of Pattern Recognition and Artificial Intelligence. 2018. - Vol. 32, no. 1. - 32 p.

14. Wang, Z. Applications of Objective Image Quality Assessment Methods [Applications Corner] [Text] / Z. Wang // IEEE Signal Processing Magazine. Nov. 2011. - Vol. 28, no. 6. - P. 137-142.

15. Image quality assessment: From error visibility to structural similarity [Text] / Z. Wang, A. Bovik, H. Sheikh, E. Simoncelli // IEEE Transactions on Image Processing. - 2004. - Vol. 13, no. 4. - P. 600-612.

16. On between-coefficient contrast masking of DCT basis functions [Text] / N. Ponomarenko, F. Silvestri, K. Egiazarian, M. Carli, J. Astola, V. Lukin // Proc. of the Third Int. Workshop on Video Processing and Quality Metrics. - Scottsdale, Arizona, USA, Jan. 2007. $-4 p$.

17. Lin, W. Perceptual visual quality metrics: $A$ survey [Text] / W. Lin, C. Jay Kuo // Journal of Visual Communication and Image Representation. - 2011. Vol. 22, no. 4. - P. 297-312.

18. Manap, $R$. Non-distortion-specific noreference image quality assessment: A survey [Text] / R. Manap, L. Shao // Information Sciences. - 2015. Vol. 301. - P. 141-160.

19. Full-reference metrics multidistortional analysis [Text] /O. Ieremeiev, V. Lukin, N. Ponomarenko, K. Egiazarian // Electronic Imaging. - 2017. - Vol. 2017, no. 13. - P. 27-35.

20. Особенности фильтрации текстурных изображений [Текст] / О. С. Рубель, А. С. Рубель, В. В. Лукин, М. К. Чобану // 18-я Международная конференция по обработке цифрровых сигналов и ее приложениям, Москва, Россия. - 2016. - C. 916-921.

21. Prediction of filtering efficiency for DCTbased image denoising [Text] / S. Abramov, S. Kriven- 
ko, A. Roenko, V. Lukin, I. Djurovic, M. Chobanu // 2nd Mediterranean Conference on Embedded Computing (MECO), Budva, Montenegro. - 2013. - P. 97-100.

22. Rubel, O. An Improved Prediction of DCTBased Filters Using Regression Analysis [Text] / O. Rubel, V. Lukin // Information and Telecommunications Sciences, Kiev, Ukraine. - 2014. - Vol. 5, no. 1. - P. 30-41.

23. Rubel, A. Regression-based Analysis of Visual Quality for Denoised Images [Text] / A. Rubel, V. Lukin // Fourth International Scientific-Practical Conference Problems of Infocommunications Science and Technology, Kharkov, Ukraine. - 2017. - P. 219-222.

24. The USC-SIPI Image Database [Electronic resource]. - Access mode: http://sipi.usc.edu/database/. Access date 8.01.2018. - Title by screen.

25. Mittal, A. No-Reference Image Quality Assessment in the Spatial Domain [Text] / A. Mittal, A. Moorthy, A. Bovik // IEEE Transactions on Image Processing. - 2012. - Vol. 21, no. 12. - P. 4695-4708.

26. No-reference image quality assessment based on spatial and spectral entropies [Text] / L. Liu, B. Liu, H. Huang, A. Bovik // Signal Processing: Image Communication. - 2014. - Vol. 29, no. 8. - P. 856-863.

27. Moorthy, A. K. A Two-Step Framework for Constructing Blind Image Quality Indices [Text] / A. K. Moorthy, A. C. Bovik // IEEE Signal Processing Letters. - 2010. - Vol. 17, no. 5. - P. 513-516.

28. Saad, M. Blind Image Quality Assessment: A Natural Scene Statistics Approach in the DCT Domain [Text] / M. Saad, A. C. Bovik, C. Charrier // IEEE Transactions on Image Processing. - 2012. - Vol. 21, no. 8. - P.3339-3352.

29. Unsupervised feature learning framework for no-reference image quality assessment [Text] / P. Ye, J. Kumar, L. Kang, D. Doermann // IEEE Conference on Computer Vision and Pattern Recognition. - 2012. P. 1098-1105.

30. Mittal, A. Making a "Completely Blind" Image Quality Analyzer [Text] / A. Mittal, R. Soundararajan, A. C. Bovik // IEEE Signal Processing Letters. - 2013. Vol. 20, no. 3. - P. 209-212.

31. Ferzli, R. A No-Reference Objective Image Sharpness Metric Based on the Notion of Just Noticeable Blur (JNB) [Text] / R. Ferzli, L. J. Karam // IEEE Transactions on Image Processing. - 2009. - Vol. 18, no. 4. - P. 717-728.

32. Blind image quality assessment by relative gradient statistics and adaboosting neural network [Text] / L. Liu, Y. Hua, Q. Zhao, H. Huang, A. Bovik// Signal Processing: Image Communication. - 2016. Vol. 40, no.1. - P. 1-15.

33. Blind Image Quality Assessment Using Joint Statistics of Gradient Magnitude and Laplacian Features [Text] / W. Xue, X. Mou, L. Zhang, A. C. Bovik,
$X$. Feng // IEEE Transactions on Image Processing. 2014. - Vol. 23, no. 11. - P. 4850-4862.

34. Moorthy, A. K. Blind Image Quality Assessment: From Natural Scene Statistics to Perceptual Quality [Text] / A. K. Moorthy, A. C. Bovik // IEEE Transactions on Image Processing. - 2011. - Vol. 20, no. 12. - P. 3350-3364.

35. Blind Image Quality Assessment Based on Multichannel Feature Fusion and Label Transfer [Text] / Q. Wu, H. Li, F. Meng, K. Ngan, B. Luo, C. Huang, B. Zeng // IEEE Transactions on Circuits and Systems for Video Technology. - 2016. - Vol. 26, no. 3. - P. 425440.

36. Using Free Energy Principle For Blind Image Quality Assessment [Text] / K. Gu, G. Zhai, X. Yang, W. Zhang // IEEE Transactions on Multimedia. - 2015. - Vol. 17, no. 1. - P. 50-63.

37. $W u, Q$. A highly efficient method for blind image quality assessment [Text] / Q. Wu, Z. Wang, H. Li // IEEE International Conference on Image Processing (ICIP). - 2015. - P. 339-343.

38. Xue, W. Learning without Human Scores for Blind Image Quality Assessment [Text] / W. Xue, L. Zhang, X. Mou // IEEE Conference on Computer Vision and Pattern Recognition. - 2013. - P. 995-1002.

39. Narvekar, N. D. A no-reference perceptual image sharpness metric based on a cumulative probability of blur detection [Text] / N. D. Narvekar, L. J. Karam // International Workshop on Quality of Multimedia Experience. - 2009. - P. 87-91.

40. Zhu, T. A no-reference objective image quality metric based on perceptually weighted local noise [Text] / T. Zhu, L. Karam // EURASIP Journal on Image and Video Processing. - 2014. - Vol. 2014, no. 1. - 8 p.

41. dipIQ: Blind Image Quality Assessment by Learning-to-Rank Discriminable Image Pairs [Text] / K. Ma, W. Liu, T. Liu, Z. Wang, D. Tao // IEEE Transactions on Image Processing. - 2017. - Vol. 26, no. 8. P. 3951-3964.

42. Blind Image Quality Assessment Based on High Order Statistics Aggregation [Text] / J. Xu, P. Ye, Q. Li, H. Du, Y. Liu, D. Doermann // IEEE Transactions on Image Processing. - 2016. - Vol. 25, no. 9. - P. 4444-4457.

43. Gong, Y. Image enhancement by gradient distribution specification [Text] / Y. Gong, I. F. Sbalzarini // 12th Asian Conference on Computer Vision, Workshop on Emerging Topics in Image Enhancement and Restoration. - Now. 2014. - 15 p.

44. Learning a no-reference quality metric for single-image super-resolution [Text] / C. Ma, C. Yang, $X$. Yang, M. Yang // Computer Vision and Image Understanding. - 2017. - Vol. 158. - P. 1-16.

45. Hybrid No-Reference Quality Metric for Singly and Multiply Distorted Images [Text] / K. Gu, G. Zhai, 
$X$. Yang, W. Zhang // IEEE Transactions on Broadcasting. - 2014. - Vol. 60, no. 3. - P. 555-567.

46. SubjectiveIQA [Electronic resource]. - Access mode: https://github.com/ViA-RiVaL/SubjectiveIQA. Access data 8.01.2018. - Tittle by screen.

\section{References}

1. Schowengerdt, R. Remote Sensing: Models and Methods for Image Processing. Academic Press Publ., 2006. 506 p.

2. Lebrun, M., Colom, M., Buades, A., Morel, J. M. Secrets of image denoising cuisine. In Acta Numerica, vol. 21, no. 1, 2012, pp. 475-576.

3. Dabov, K., Foi, A., Katkovnik, V., Egiazarian, $\mathrm{K}$. Image denoising by sparse $3 \mathrm{D}$ transform-domain collaborative filtering. IEEE Transactions on Image Processing, vol. 16, no. 8, 2007, pp. 2080-2095.

4. Lukin, V., Oktem, R., Ponomarenko, N., Egiazarian, K. Image filtering based on discrete cosine transform. Telecommunications and Radio Engineering, vol. 66, no. 18, 2007, pp. 1685-1701.

5. Pižurica, A. Image Denoising Algorithms: From Wavelet Shrinkage to Nonlocal Collaborative Filtering. Wiley Encyclopedia of Electrical and Electronics Engineering, 2017. $17 \mathrm{p}$.

6. Zhang, K., Zuo, W., Chen, Y., Meng, D., Zhang, L. Beyond a Gaussian Denoiser: Residual Learning of Deep CNN for Image Denoising. IEEE Transactions on Image Processing, vol. 26, no. 7, 2017, pp. 3142-3155.

7. Choi, S., Isidoro, J., Getreuer, P., MIlanfar, P. Fast, Trainable, Multiscale Denoising. ArXiv preprint arXiv: $1802.06130,2018.5 \mathrm{p}$.

8. Zheng, K., Wang, Z. Perceptual evaluation of image denoising algorithms. Asilomar Conference on Signals, Systems and Computers, Pacific Grove, CA, 2013, pp. 1351-1355.

9. Ponomarenko, N., Jin, L., Ieremeiev, O., Lukin, V., Egiazarian, K., Astola, J., Vozel, B., Chehdi, K., Carli, M., Battisti, F., Jay Kuo, C.-C. Image database TID2013: Peculiarities, results and perspectives. Signal Processing: Image Communication, vol. 30, 2015, pp. 57-77.

10. Egiazarian, K., Ponomarenko, M., Lukin, V., Ieremeiev, O. Statistical evaluation of visual quality metrics for image denoising. ArXiv preprint arXiv: 1711.00693, 2017. 5 p.

11. Rubel, O., Ponomarenko, N., Lukin, V., Astola, J., Egiazarian, K. HVS-based local analysis of denoising efficiency for DCT-based filters. Second International Scientific-Practical Conference Problems of Infocommunications Science and Technology (PIC S\&T), Kharkiv, Ukraine, Oct. 2015, pp. 189-192.

12. Rubel, A., Rubel, O., Lukin, V. Analysis of visual quality for denoised images. 14th International
Conference The Experience of Designing and Application of CAD Systems in Microelectronics (CADSM), Lviv, Ukraine, 2017, pp. 92-96.

13. Rubel, O., Lukin, V., Abramov, S., Vozel, B., Pogrebnyak, O., Egiazarian, K. Is Texture Denoising Efficiency Predictable? International Journal of Pattern Recognition and Artificial Intelligence, vol. 32, no. 1, 2018. 32 p.

14. Wang, Z. Applications of Objective Image Quality Assessment Methods [Applications Corner]. IEEE Signal Processing Magazine, vol. 28, no. 6, Nov. 2011, pp. 137-142.

15. Wang, Z., Bovik, A., Sheikh, H., Simoncelli, E. Image quality assessment: From error visibility to structural similarity. IEEE Transactions on Image Processing, vol. 13, no. 4, 2004, pp. 600-612.

16. Ponomarenko, N., Silvestri, F., Egiazarian, K., Carli, M., Astola, J., Lukin, V. On between-coefficient contrast masking of DCT basis functions. Proc. of the Third Int. Workshop on Video Processing and Quality Metrics, Scottsdale, Arizona, USA, Jan. 2007. 4 p.

17. Lin, W., Jay Kuo, C. Perceptual visual quality metrics: A survey. Journal of Visual Communication and Image Representation, vol. 22, no. 4, 2011, pp. 297312.

18. Manap, R., Shao, L. Non-distortion-specific no-reference image quality assessment: A survey. Information Sciences, vol. 301, 2015, pp. 141-160.

19. Ieremeiev, O., Lukin, V., Ponomarenko, N., Egiazarian, K. Full-reference metrics multidistortional analysis. Electronic Imaging, vol. 2017, no. 13, 2017 , pp. 27-35.

20. Rubel', O. S., Rubel', A. S., Lukyn, V. V., Chobanu, M. K. Osobennosty fyl'tratsyy teksturnykh yzobrazhenyy [Peculiarities of texture image filtering]. 18-ya Mezhdunarodnaya konferentsyya po obrabotke tsyfrovykh syhnalov y ee prylozhenyyam [International Conference on Digital Signal Processing and its Applications], Moskva, Rossyya, no. 2, 2016, pp. 916-921.

21. Abramov, S., Krivenko, S., Roenko, A., Lukin, V., Djurovic, I., Chobanu, M. Prediction of filtering efficiency for DCT-based image denoising. 2nd Mediterranean Conference on Embedded Computing (MECO), Budva, Montenegro, 2013, pp. 97-100.

22. Rubel, O., Lukin, V. An Improved Prediction of DCT-Based Filters Using Regression Analysis. Information and Telecommunications Sciences, Kiev, Ukraine, vol. 5, no. 1, 2014, pp. 30-41.

23. Rubel, A., Lukin, V. Regression-based Analysis of Visual Quality for Denoised Images. Fourth International Scientific-Practical Conference Problems of Infocommunications Science and Technology (PIC S\&T), Kharkov, Ukraine, 2017, pp. 219-222.

24. The USC-SIPI Image Database. Available at: http://sipi.usc.edu/database/ (accessed 8.01.2018). 
25. Mittal, A., Moorthy, A., Bovik, A. NoReference Image Quality Assessment in the Spatial Domain. IEEE Transactions on Image Processing, vol. 21, no. 12, 2012, pp. 4695-4708.

26. Liu, L., Liu, B., Huang, H., Bovik, A. Noreference image quality assessment based on spatial and spectral entropies. Signal Processing: Image Communication, vol. 29, no. 8, 2014, pp. 856-863.

27. Moorthy, A. K., Bovik, A. C. A Two-Step Framework for Constructing Blind Image Quality Indices. IEEE Signal Processing Letters, vol. 17, no. 5, 2010, pp. 513-516.

28. Saad, M., Bovik, A. C., Charrier, C. Blind Image Quality Assessment: A Natural Scene Statistics Approach in the DCT Domain. IEEE Transactions on Image Processing, vol. 21, no. 8, 2012, pp. 3339-3352.

29. Ye, P., Kumar, J., Kang, L., Doermann, D. Unsupervised feature learning framework for no-reference image quality assessment. IEEE Conference on Computer Vision and Pattern Recognition, 2012, pp. 10981105.

30. Mittal, A., Soundararajan, R., Bovik, A. Making a "Completely Blind" Image Quality Analyzer. IEEE Signal Processing Letters, vol. 20, no. 3, 2013, pp. 209-2212.

31. Ferzli, R., Karam, L. A No-Reference Objective Image Sharpness Metric Based on the Notion of Just Noticeable Blur (JNB). IEEE Transactions on Image Processing, vol. 18, no. 4, 2009, pp. 717-728.

32. Liu, L., Hua, Y., Zhao, Q., Huang, H., Bovik, A. Blind image quality assessment by relative gradient statistics and adaboosting neural network. Signal Processing: Image Communication, vol. 40, no.1, 2016, pp. $1-15$.

33. Xue, W., Mou, X., Zhang, L., Bovik, A., Feng, X. Blind Image Quality Assessment Using Joint Statistics of Gradient Magnitude and Laplacian Features. IEEE Transactions on Image Processing, vol. 23, no. 11, 2014, pp. 4850-4862.

34. Moorthy, A. K., Bovik, A. C. Blind Image Quality Assessment: From Natural Scene Statistics to Perceptual Quality. IEEE Transactions on Image Processing, vol. 20, no. 12, 2011, pp. 3350-3364.
35. Wu, Q., Li, H., Meng, F., Ngan, K., Luo, B., Huang, C., Zeng, B. Blind Image Quality Assessment Based on Multichannel Feature Fusion and Label Transfer. IEEE Transactions on Circuits and Systems for Video Technology, vol. 26, no. 3, 2016, pp. 425-440.

36. Gu, K., Zhai, G., Yang, X., Zhang, W. Using Free Energy Principle For Blind Image Quality Assessment. IEEE Transactions on Multimedia, vol. 17, no. 1, 2015, pp. 50-63.

37. Wu, Q., Wang, Z., Li, H. A highly efficient method for blind image quality assessment. IEEE International Conference on Image Processing (ICIP), 2015, pp. 339-343.

38. Xue, W., Zhang, L., Mou, X. Learning without Human Scores for Blind Image Quality Assessment. IEEE Conference on Computer Vision and Pattern Recognition, 2013, pp. 995-1002.

39. Narvekar, N. D., Karam, L. J. A no-reference perceptual image sharpness metric based on a cumulative probability of blur. International Workshop on Quality of Multimedia Experience, 2009, pp. 87-91.

40. Zhu, T., Karam, L. A no-reference objective image quality metric based on perceptually weighted local noise. EURASIP Journal on Image and Video Processing, vol. 2014, no. 1, 2014. 8 p.

41. Ma, K., Liu, W., Liu, T., Wang, Z., Tao, D. dipIQ: Blind Image Quality Assessment by Learning-toRank Discriminable Image Pairs. IEEE Transactions on Image Processing, vol. 26, no. 8, 2017, pp. 3951-3964.

42. Xu, J., Ye, P., Li, Q., Du, H., Liu, Y., Doermann, D. Blind Image Quality Assessment Based on High Order Statistics Aggregation. IEEE Trans. on Image Processing, vol. 25, no. 9, 2016, pp. 4444-4457.

43. Gong, Y., Sbalzarini, I. F. Image enhancement by gradient distribution specification. 12th Asian Conference on Computer Vision, Workshop on Emerging Topics in Image Enhancement and Restoration, Now. 2014. $15 \mathrm{p}$.

44. Ma, C., Yang, C., Yang, X., Yang, M. Learning a no-reference quality metric for single-image super-resolution. Computer Vision and Image Understanding, vol. 158, 2017, pp. 1-16.

45. Gu, K., Zhai, G., Yang, X., Zhang, W. Hybrid No-Reference Quality Metric for Singly and Multiply Distorted Images. IEEE Transactions on Broadcasting, vol. 60 , no. 3, 2014, pp. 555-567.

46. SubjectiveIQA. Available at: https://github. com/ViA-RiVaL/SubjectiveIQA (accessed 8.01.2018). 


\title{
АНАЛІЗ І ПРОГНОЗУВАННЯ ЕФЕКТИВНОСТІ ФІЛЬТРАЦЇ̈ З ВИКОРИСТАННЯМ БЕЗЕТАЛОННИХ МІР ВІЗУАЛЬНОЇ ЯКОСТІ ЗОБРАЖЕНЬ
}

\author{
А. С. Рубель, В. В. Лукін
}

Проведено аналіз ефективності та доцільності застосування фільтрації з використанням безеталонних мір візуальної якості зображень. Також розглянуто можливі способи прогнозування суб'єктивних оцінок візуальної якості зображень після застосування фільтрації на основі одного або кількох вхідних параметрів. Аналіз виконано для ДКП і BM3D-фільтрів. За допомогою спеціалізованої бази тестових зображень SubjectiveIQA проаналізовано відповідність сприйняттю людини існуючих безеталонних мір візуальної якості для відфільтрованих зображень. Показано, що коефіцієнт рангової кореляції Спірмена між значеннями безеталонних мір та суб'єктивними оцінками не перевищує $0,7$.

Ключові слова: ефективність фільтрації, безеталонні міри візуальної якості зображень, прогнозування, ДКП фільтр, BM3D, адитивні завади, суб'єктивний експеримент.

\section{ANALYSIS AND PREDICTION OF FILTERING EFFICIENCY USING NO-REFERENCE IMAGE VISUAL QUALITY METRICS}

\section{A. S. Rubel, V. V. Lukin}

Images are subject to noise during acquisition, transmission and processing. Image denoising is highly desirable, not only to provide better visual quality, but also to improve performance of the subsequent operations such as compression, segmentation, classification, object detection and recognition. In the past decades, a large number of image denoising algorithms has been developed, ranging from simple linear methods to complex methods based on similar blocks search and deep convolutional neural networks. However, most of existing denoising techniques have a tendency to oversmooth image edges, fine details and textures. Thus, there are cases when noise reduction leads to loss of image features and filtering does not produce better visual quality. According to this, it is very important to evaluate denoising result and hence to undertake a decision whether denoising is expedient. Despite the fact that image denoising has been one of the most active research areas, only a little work has been dedicated to visual quality evaluation for denoised images. There are many approaches and metrics to characterize image quality, but adequateness of these metrics is of question. Existing image quality metrics, especially no-reference ones, have not been thoroughly studies for image denoising. In terms of using visual quality metrics, it is usually supposed that the higher the improvement for a given metric, the better visual quality for denoised image. However, there are situations when denoising does not result in visual quality enhancement, especially for texture images. Thus, it would be desirable to predict human subjective evaluation for denoised image. Then, this information will clarify when denoising can be expedient. The purpose of this paper is to give analysis of denoising expedience using no-reference (NR) image quality metrics. In addition, this work considers possible ways to predict human subjective evaluation of denoised images based on several input parameters. More in details, two denoising techniques, namely the standard sliding window DCT filter and the BM3D filter have been considered. Using a specialized database of test images SubjectiveIQA, performance evaluation of existing state-of-the-art objective no-reference quality metrics for denoised images is carried out.

Keywords: denoising efficiency, no-reference image visual quality metrics, prediction, DCT filter, BM3D, additive noise, subjective experiment.

Рубель Андрей Сергеевич - аспирант каф. информационно-коммуникационных технологий им. А. А. Зеленского, Национальный аэрокосмический университет им. Н. Е. Жуковского «Харьковский авиационный институт», Харьков, Украина, e-mail: rubel.andrew@gmail.com.

Лукин Владимир Васильевич - д-р техн. наук, проф., заведующий каф. информационнокоммуникационных технологий им. А. А. Зеленского, Национальный аэрокосмический университет им. Н. Е. Жуковского «Харьковский авиационный институт», Харьков, Украина, e-mail: lukin@ai.kharkov.com.

Rubel Andrej Sergeevich - PhD student of Dept. of Information and communication technologies named after A.A. Zelensky, National Aerospace University "Kharkiv Aviation Institute", Kharkov, Ukraine, e-mail: rubel.andrew@gmail.com.

Lukin Vladimir Vasilyevich - Doctor of Technical Science, Professor, Head of Dept. of Information and communication technologies named after A.A. Zelensky, National Aerospace University "Kharkiv Aviation Institute", Kharkov, Ukraine, e-mail: lukin@ai.kharkov.com. 